\title{
ESTUDIO DINÁMICO DEL COMPORTAMIENTO DE UN ROBOT ÁPODO MODULAR E HIPER-REDUNDANTE
}

\author{
José L. González, Javier P. Turiel, Juan C. Fraile, María A. Pérez, Ricardo Martín. \\ ITAP- Instituto de las Tecnologías Avanzadas de la Producción, Universidad de Valladolid. \\ jossan@eii.uva.es ; \{turiel, jcfraile, marper\}@eii.uva.es
}

\begin{abstract}
Resumen
La aplicación de la robótica en la medicina se ha incrementado de manera significativa en los últimos años. Sin embargo todavía existen procedimientos de diagnóstico, como la endoscopia, en los que existen limitaciones importantes. Esta técnica tiene todavía un gran margen de mejora siendo de gran interés el avance de sus capacidades de acceso y maniobrabilidad, la óptica, la calidad de imagen y el desarrollo de nuevos materiales y métodos.

En el presente trabajo se analizará el comportamiento físico, en especial el aspecto dinámico que afecta a un robot de este tipo con el objetivo de comprobar las características de los distintos componentes que lo forman y tener la opción de variarlos buscando la optimización de cada uno de ellos
\end{abstract}

Palabras Clave: locomoción ápoda, robot modular, robot híper-redundante (HRR), endoscopia, análisis dinámico.

\section{INTRODUCCIÓN}

La robótica ha asumido un papel de liderazgo en el actual desarrollo socioeconómico. En particular, su uso en la medicina se ha incrementado de manera exponencial en los últimos años, principalmente en las áreas de cirugía, rehabilitación, diagnóstico, tratamiento de enfermedades, aporte de medicamentos, prótesis y asistencia remota a los pacientes. El uso de sistemas robóticos ha mejorado de forma notable las prestaciones con respecto al riesgo de infección y el tiempo de convalecencia. Además, se ha convertido en una valiosa ayuda en cuanto a la monitorización del paciente [1][6].

Sin embargo, todavía existen procedimientos de diagnóstico, como la endoscopia, que son poco tolerados por los pacientes, por causar lesiones y molestias. Esta técnica tiene todavía margen de mejora siendo de gran interés el avance de sus capacidades de acceso, la óptica, la calidad de imagen y el desarrollo de nuevos materiales y métodos. Por lo tanto, una de las áreas de interés de investigación es el uso de dispositivos robóticos como endoscopios autónomos y semiautónomos que pueden aportar ventajas debido a su capacidad de movimiento a lo largo de la cavidad para una observación más detallada, y también pueden reducir al mínimo el contacto con los tejidos y permitiendo una mejor orientación del instrumento, superando con ello las limitaciones del endoscopio tradicional [8].

En este artículo se estudiará la dinámica de un robot ápodo modular e híper-redundante, en función de los esfuerzos que le afecten.

\section{ROBOT ÁPODO, MODULAR E HÍPER-REDUNDANTE}

Los robos ápodos no utilizan ruedas ni patas, sino movimientos de su propio cuerpo para generar desplazamiento. El accionamiento independiente de cada articulación da lugar a una estructura cinemática híper-redundante [5][10], con un elevado número de grados de libertad, lo que le permite adoptar múltiples configuraciones. Un robot es redundante si el número de grados de libertad es mayor que el valor mínimo para su funcionamiento. La característica híperredundante de este tipo de robots proporciona una ventaja para el desplazamiento y la maniobrabilidad en ambientes irregulares. Adicionalmente, tienen una sección transversal mucho menor lo cual les permite moverse por espacios reducidos [5].

El estado de la técnica de los dispositivos desarrollados para procedimientos de endoscopia muestra que la prioridad principal es realizar un sistema con una acción de locomoción interna, capaz de avanzar en un entorno complejo y que reduzca los riesgos de perforación [2]. El sistema ideal debe ser seguro, lo menos invasivo posible y conseguir una alta precisión diagnóstica. Por lo tanto, un dispositivo muy flexible y suave, que deforma suavemente y sólo localmente el tejido humano, representa la solución óptima [2][9].

Para los procedimientos endoscópicos, dos de los requisitos claves son la adecuada articulación del robot, para asegurar que no se dañe los tejidos durante la inspección, y un amplio ángulo de exploración visual [4][5]. También es muy importante la elección 
del material y los actuadores, el dispositivo debe estar construido con materiales biocompatibles con el cuerpo humano, de tal forma que no se produzcan complicaciones durante la operación ni efecto adverso a posteriori [3].

\section{ESTUDIO DINÁMICO}

En este apartado analizan los distintos esfuerzos a los que se somete a cada módulo del robot. Se definirá cada tipo de esfuerzo o fuerza así como su origen y punto aplicación con el objeto de resolver el sistema dinámico que permita obtener información sobre las características mecánicas de distintos componentes del módulo. Uno de los pricipales objetivos será conocer el valor de la fuerza que debe suministrar el actuador comprobando si cumple con los requisitos y, en caso negativo, elegir otro actuador que aporte la fuerza requerida a la velocidad necesaria para el movimiento del sistema. Otro objetivo es la verificacion del eje permitiendo conocer si su rigidez estructural es suficiente para aguantar los distintos esfuerzos a los que se verá sometido.

\subsection{MODELADO DINÁMICO}

En este apartado se definirá la estructura, el funcionamiento, los posibles movimientos y las variables del robot ápodo híper-redundante

\subsubsection{Definición del robot ápodo}

El robot se mueve, partiendo de un extremo fijo, que se denomina base, de forma que el extremo libre se posicione lo más cerca posible al punto deseado y con la orientación requerida. Se cuenta con un número variable de módulos formados por placas circulares sobre las que se colocan los actuadores.

Se ha elegido un tipo de actuador [7][11](figura 1) basado en láminas IPMC que, pueden tener características adecuadas para su uso en endoscopia, ya que son de pequeño tamaño, y se necesita para su activación una intensidad de corriente muy baja.

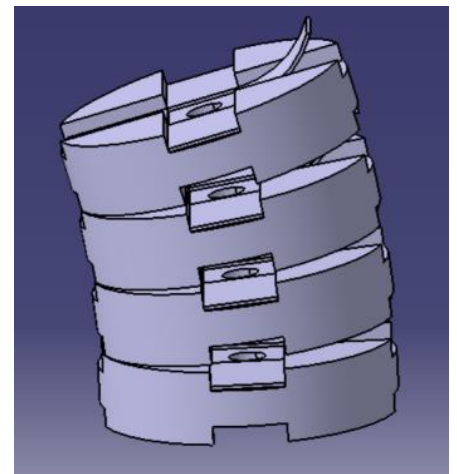

Figura 1: Diseño propuesto [7][11].
Consta de una serie de módulos, cada uno formado por dos placas como la mostrada en la figura 2, comunes para dos módulos consecutivos, y cuatro actuadores que se ubicarían en los huecos mostrados en la figura.

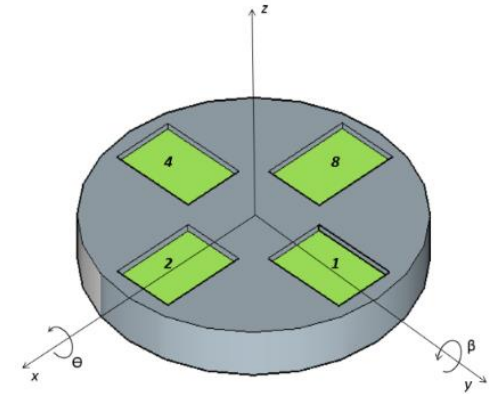

Figura 2: Diseño de una placa y ejes de referencia.

Las placas se suponen cilíndricas, de dimensiones y material conocido. Los actuadores, la parte del eje correspondiente, así como el recubrimiento exterior si lo hubiese, se considerarán de pesos despreciables con respecto al de la placa. En la figura 2 se muestran los ejes y las variables de giro asociadas.

La primera placa contendrá el anclaje del robot, que servirá como base de referencia del robot, mientras que en la última placa se instalará una cámara que servirá para captar las imágenes de la endoscopia. Las placas se interconectan mediante eje central elástico.

\subsubsection{Características geométricas}

En la figura 3 se muestran los parámetros geométricos principales. Se considera un sistema de referencia en base solidaria a la placa que se estudia, con origen en su centro de masas, al que llamaremos $\mathrm{O}_{\mathrm{m}}$. El subíndice ' $\mathrm{m}$ ' nos indicara la placa en la que nos encontramos.

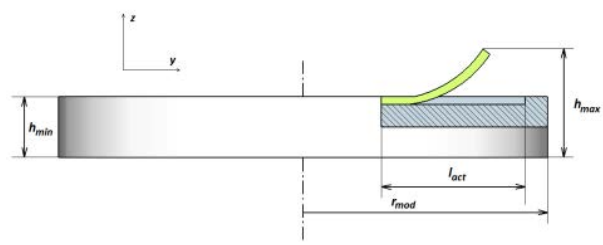

Figura 3: Parámetros geométricos de la placa.

Según se muestra en la figura 2 , se definen $\theta$ y $\beta$ como los posibles giros de la placa sobre los ejes ' $x$ ' e ' $y$ ', respectivamente. Añadiendo el posible movimiento longitudinal sobre el eje ' $z$ ', esos serán los tres tipos de movimiento que se considerarán según la configuración de cada módulo.

\subsubsection{Características funcionales}

Se tiene un sistema con cuatro actuadores por cada módulo como se muestra en las figuras 1 y 2 . Estos actuadores asumen dos estados: activo y neutro o inactivo, como se muestra en la figura 4. 
Esto permite obtener, por cada módulo, cuatro configuraciones de giro (casos 1, 2, 4 y 8) con un actuador activo en cada caso y dos configuraciones longitudinales, la configuración 0 (ningún actuador activo) y la 15 (todos activos)

Para este estudio dinámico se ha considerado el uso de actuadores IPMC (Ionic Polimer-Metal Composites). Estos actuadores son activados mediante una tensión suministrada en sus caras laterales. Este diferencial de tensión lleva al actuador a activarse, curvándose para alcanzar la placa superior y ejercer así su fuerza sobre la misma. Como ventajas de estos actuadores están la baja tensión necesaria, que aunque puede aumentarse para conseguir mayor respuesta se sitúa sobre los $2 \mathrm{~V}$. Además permite ejercer unas fuerzas que a priori suponemos suficientes en función de la tensión suministrada. Se estudiará si son suficientes para los objetivos planteados. En contraposición, la rapidez de activación es menor respecto a otros tipos de actuadores.

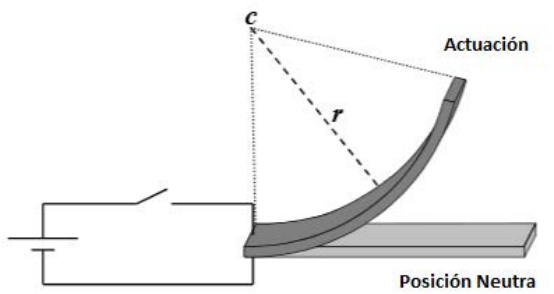

Figura 4: Posibles estados de actuación de una lámina IPMC.

La fuerza de accionamiento está directamente relacionada con las dimensiones del actuador y el voltaje aplicado (figura 5).

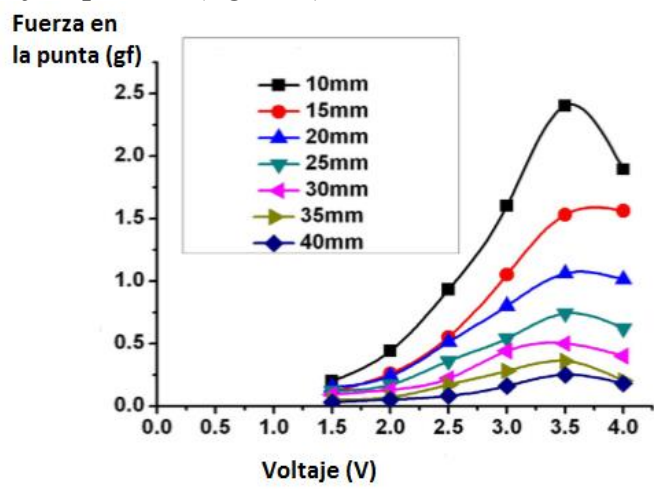

Figura 5: Relación fuerza de salida-voltaje aplicado en un actuador IPMC Nafion ${ }^{\circledR} 117$ de $200 \mu \mathrm{m}$ de espesor y longitud variable.

\subsubsection{Variables y movimientos correspondientes}

Se usan tres variables para definir el movimiento entre el par de placas que conforman cada módulo: dos variables angulares, $\theta$ y $\beta$, y una longitudinal, h. Estas tres variables permiten definir la posición relativa de una placa respecto a la anterior.
Ángulo theta (⿻): giro de una placa con respecto a la anterior en el eje ' $\mathrm{x}$ ', presente en las configuraciones 2 y 8 . Para estas dos configuraciones estáticas tendrá un valor determinado y fijo, dependiente del actuador. Suponiendo que el actuador es capaz de proporcionar una altura $\left(h_{\mathrm{act}}\right)$ igual a la mitad de su longitud:

$$
h_{a c t}=\frac{l_{a c t}}{2}
$$

el ángulo $\theta$ será:

$$
\theta=\arctan \left(\frac{h_{a c t}}{\mathrm{l}^{*}}\right)
$$

donde $1^{*}$ corresponde a la base del triángulo que forman las dos placas que separa el actuador.

Ángulo beta ( $(\boldsymbol{\theta})$ : giro de una placa con respecto a la anterior en el eje ' $y$ ', presente en las configuraciones 1 y 4. Para estas dos configuraciones tendrá un valor fijo, dependiente del actuador:

$$
\beta=\arctan \left(\frac{h_{a c t}}{\mathrm{l}^{*}}\right)
$$

Altura (h): separación en el eje ' $z$ ' de dos placas contiguas. Variable principal de las configuraciones 0 y 15 , con valores máximos y mínimos $\mathrm{h}_{\max } \mathrm{y} \mathrm{h}_{\text {min }}$ respectivamente

\subsection{DIAGRAMA DE FUERZAS}

Para realizar este estudio, se aborda el tratamiento de cada placa de forma aislada, considerando que el resto de placas superiores forman un sólido rígido con ella. Para ello se define cada una de las fuerzas y momentos que la afectan. En la figura 6 se muestra el diagrama de fuerzas y momentos actuando en el caso del giro en ' $z$ ', $\theta$, caso en un actuador está activo. Se muestran todos los esfuerzos que se considerarán en el estudio. Se sitúa también la placa anterior $(\mathrm{m}-1)$ para tenerla como referencia.

Las placas con centros $\mathrm{O}_{\mathrm{m}}, \mathrm{O}_{\mathrm{m}+1}$ y $\mathrm{O}_{\mathrm{m}+2}$ forman el sólido rígido a analizar.

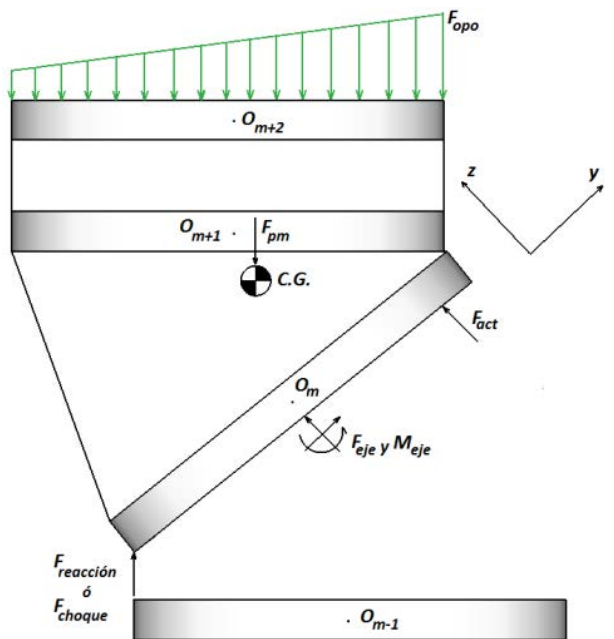

Figura 6: Diagrama de fuerzas y momentos de la placa para el caso del actuador 3 activo. 
- $\quad \mathrm{F}_{\text {opo }}$ : fuerza dada por la oposición del medio exterior al movimiento del robot.

- $F_{\text {act: }}$ fuerza debida a los actuadores.

- $F_{\text {reacc }} / F_{\text {choque }}$ fuerza que ejerce la placa anterior sobre la estudiada, de reacción cuando hayan estado en contacto en todo el movimiento, y de choque cuando pasen de no tocarse a hacer contacto.

- $F_{p m}$ : fuerza equivalente del peso del bloque formado por el módulo estudiado $y$ posteriores.

- $\mathrm{F}_{\text {eje }} / \mathrm{M}_{\text {eje: }}$ fuerza o momento debido a la actuación del eje.

- C.G.: centro de masas del conjunto.

Para su resolución, el problema se plantea con dos sistemas de ecuaciones. El primero, dado por el teorema de conservación de momento cinético, del que se podrán obtener 3 ecuaciones. El segundo sistema nos lo aporta la segunda ley de Newton y de nuevo dará otras 3 ecuaciones.

\subsection{FUERZAS Y MOMENTOS}

A continuación se definen cada una de las fuerzas y los respectivos momentos que afectarán a cada placa de nuestro robot. Entre ellas habrá efectos que se han de contrarrestar para conseguir el movimiento, como es el caso del peso que puedan tener los módulos siguientes al estudiado o la oposición al movimiento que ejerce el fluido exterior en el que se encuentra el robot. Otros efectos, sin embargo, son los que utiliza el robot para conseguir su objetivo. Como principales se tendrá el efecto que ejercen los actuadores para separar las placas contiguas o el aplicado por el eje, que suministra la fuerza para acercar las placas y mantenerlas en el mismo plano. Estos dos efectos son los que se requiere conocer y los que se podrían cambiar mediante variaciones en los componentes o en los materiales que los forman.

\subsubsection{Peso de los módulos superiores}

Una de las resistencias que deberán superar los actuadores será el peso del módulo estudiado y de todos los módulos siguientes. Se suponen los módulos de la parte en voladizo como un bloque sólido anclado a la placa $m$ estudiada.

Para el cálculo de la posición del centro de gravedad (C.G.), suponiendo que cada módulo es idéntico al resto, y que cada una de las configuraciones tiene unos desplazamientos fijos, para calcular el centro de masas se toman unos vectores de avance, $\left(\overrightarrow{O_{l} O_{l+1} \text { caso }_{J}}\right)$, que indican la posición relativa del centro de una placa, $\mathrm{O}_{\mathrm{i}+1}$, con respecto a la placa anterior, en base solidaria a esta última placa. Dicho vector depende de la configuración del módulo que conforman dichas placas. Partiendo del punto $O_{m}$, y conociendo la configuración de los módulos componentes del solido rígido, se procede placa a placa a obtener cada centro. Sería:

$$
\begin{aligned}
& \overrightarrow{\text { c.d.g. }}_{\text {modulo } n}={\overrightarrow{O_{m} O_{m+1} \text { caso }_{J}}}+ \\
& \quad+\left(M_{\text {transf } m+1}\right) \cdot \overrightarrow{O_{m+1} O_{m+2} \text { caso }_{J}}+ \\
& \ldots+\left(M_{\text {transf } m+1}\right) \cdot \ldots \cdot\left(M_{\text {transf } n-1}\right) \cdot \overrightarrow{O_{n-1} O_{n_{\text {caso }}}}
\end{aligned}
$$

Siendo ' $n$ ' la última placa. Cambiando consecuentemente de base cada vector avance con ( $\left.M_{\text {transf } i}\right)$ hacia la referencia solidaria a ' $\mathrm{m}$ ' $\mathrm{y}$ sumándolos se obtiene el C.G. de cada placa superior con respecto a $\mathrm{O}_{\mathrm{m}}$ :, donde:

- $\overrightarrow{O_{l} O_{l+1} \text { caso }_{J}}$.- Posición relativa de la placa de centro $\mathrm{O}_{\mathrm{i}+1}$ con respecto a la anterior en la referencia de esta última.

- Mtransf - $^{-}$Matriz que cambia la base de los vectores de avance a la referencia de la placa anterior. Al tener dos giros, en $\theta$ y en $\beta$, tendremos dos matrices:

$$
\begin{aligned}
M_{\text {transf } x}(\theta) & =\left(\begin{array}{ccc}
1 & 0 & 0 \\
0 & \cos (\theta) & \sin (\theta) \\
0 & -\sin (\theta) & \cos (\theta)
\end{array}\right) \\
M_{\text {transf } y}(\beta) & =\left(\begin{array}{ccc}
\cos (\beta) & 0 & -\sin (\beta) \\
0 & 1 & 0 \\
\sin (\beta) & 0 & \cos (\beta)
\end{array}\right)
\end{aligned}
$$

De la misma forma es necesario conocer la orientación que tiene el vector de la gravedad en la referencia solidaria a la placa en la que se trabaja. Para ello se toma el vector de gravedad inicial, en el sistema de referencia tierra, en el que está la base del robot:

$$
\vec{g}=\left(\begin{array}{lll}
0 & 0 & -g
\end{array}\right)^{T}
$$

Dicho vector, mediante las matrices correspondientes, será transformado a la referencia utilizada. Hay que considerar que puede variar en función de las variables estudiadas y por lo tanto no será una constante:

$$
\vec{g}_{\text {ref solidaria }}=M_{\text {transf } m} \cdot \ldots \cdot M_{\text {transf } 1} \cdot \vec{g}
$$

Como resultado obtenemos el vector de fuerzas equivalentes:

$$
\vec{F}_{p m}=n^{\underline{o}}{ }_{\text {mods }} m_{\text {mod }} \vec{g}_{\text {ref solidaria }}
$$

donde:

$n^{\underline{0}}{ }_{\text {mods }}$.- Número de módulos posteriores

$m_{\text {mod }}$ - Masa de cada uno de esos módulos 


\subsubsection{Efecto de los actuadores}

El actuador funciona ejerciendo una fuerza perpendicular a la superficie que empuja, según la figura 7 .

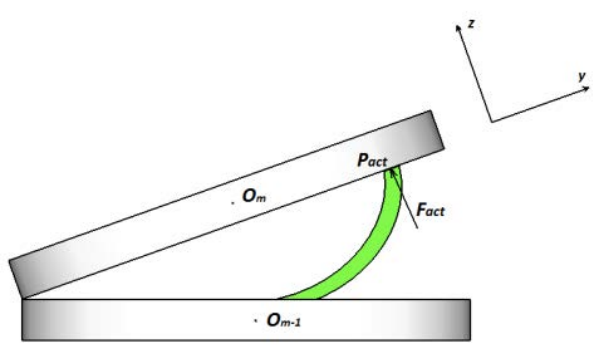

Figura 7: Fuerza y punto de aplicación de la misma por el efecto de un actuador.

Para cada actuador, la fuerza será:

$$
\overrightarrow{F_{\text {act }}}=F_{\text {act }} \overrightarrow{n_{m}}
$$

siendo $\overrightarrow{n_{m}}$ el vector unitario perpendicular a la superficie en contacto de la placa $\mathrm{m}$.

El momento respecto a C.G. correspondiente para cada actuador:

$$
\overrightarrow{M_{A c t}}=\overrightarrow{C . G . P_{a c t}} \wedge F_{a c t} \overrightarrow{n_{m}}
$$

donde:

$$
\begin{aligned}
& \text { - } \overrightarrow{C . G . P_{a c t}}=\overrightarrow{C . G . O_{m}}+\overrightarrow{O_{m} P_{a c t}} \\
& \text { - } \overrightarrow{\text { C.G.O. }} \text { obtenido en (4) } \\
& -\overrightarrow{O_{m} P_{a c t}}=\left(\begin{array}{lll} 
\pm d_{a c t} & 0 & 0
\end{array}\right)^{T} \\
& \left(0 \pm d_{a c t} 0\right)^{T} \text { según el caso }
\end{aligned}
$$

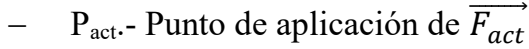

$$
\begin{aligned}
& \text { - } \mathrm{d}_{\text {act. }} \text { - Distancia del centro de la placa al } \\
& \text { punto de aplicación del actuador. }
\end{aligned}
$$

\subsubsection{Efecto del eje central}

Se considera que el eje interior de cada módulo empotrado está empotrado, en ambos extremos, a los centros de cada placa. Así, el eje pasa a ser la estructura interna principal de aguante y reacción (figura 8).
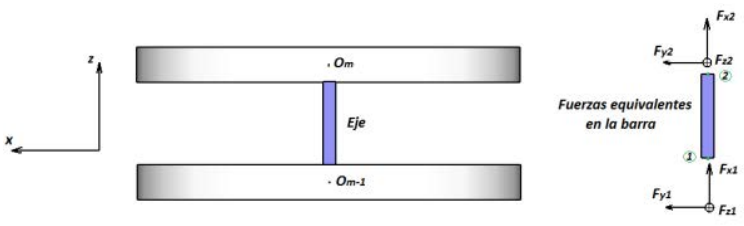

Figura 8: Disposición del eje entre placas y distribución de las fuerzas existentes en los extremos de la misma.

Tratando el eje como una barra, se aproxima su estudio utilizando el método directo de rigidez, suponiendo válido la magnitud de los desplazamientos. Para su definición, se define la matriz de rigidez local de una barra bi-empotrada.

A la hora de expresar las ecuaciones, conviene separar en un vector los momentos y en otro las fuerzas. El vector de fuerzas será:

$$
\begin{aligned}
& \left(\begin{array}{l}
F x 2 \\
F y 2 \\
F z 2
\end{array}\right)=\left[\begin{array}{cccccc}
\frac{E A}{L} & 0 & 0 & 0 & 0 & 0 \\
0 & \frac{12 E I_{z}}{L^{3}} & 0 & 0 & 0 & \frac{-6 E I_{z}}{L^{2}} \\
0 & 0 & \frac{12 E I_{y}}{L^{3}} & 0 & \frac{6 E I_{y}}{L^{2}} & 0
\end{array}\right] \times\left(\begin{array}{c}
U z \\
U x \\
U y \\
0 \\
\theta \\
\beta
\end{array}\right)= \\
& =\underset{F_{\text {eje int }}}{\longrightarrow}=\left(\begin{array}{c}
F z \\
F x \\
F y
\end{array}\right)
\end{aligned}
$$

Donde E es el módulo de Young del material del eje; $\mathrm{G}$ el módulo elástico trasversal del material del eje; $\mathrm{L}$ la longitud del eje; $\mathrm{I}_{\mathrm{p}}$.el momento de inercia torsional del eje; $I_{x}, I_{y}, I_{z}$. los segundos momentos de inercia o momentos de inercia de área del eje; $\mathrm{y} \mathrm{F}_{\mathrm{x}}, \mathrm{F}_{\mathrm{y}}, \mathrm{y} \mathrm{F}_{\mathrm{z}}$ las fuerzas expresadas en nuestra referencia solidaria a la placa. Esta es la fuerza ejercida en el eje. Por el principio de acción y reacción la buscada en la placa será la misma de sentido contrario.

$$
\overrightarrow{F_{\text {eje }}}=-\overrightarrow{F_{\text {eje } \mathrm{int}}}
$$

De igual manera se obtiene $\overrightarrow{M_{\text {ejeınt }}}=\left(M_{x} M_{y} M_{z}\right)$

Expresados $\mathrm{M}_{\mathrm{x}}, \mathrm{M}_{\mathrm{y}} \mathrm{y} \mathrm{M}_{\mathrm{z}}$ en la referencia solidaria. De esta forma, el momento respecto a C.G. queda:

$$
\overrightarrow{M_{\text {eje }}}=\overrightarrow{C . G . P_{\text {eje }}} \wedge\left(\overrightarrow{F_{\text {eje }}}\right)-\overrightarrow{M_{\text {ejernt }}}
$$

donde $\overrightarrow{C . G . P_{e j e}}=\overrightarrow{C . G . O_{m}}+\overrightarrow{O_{m} P_{e j e}}$, y $P_{e j e}$ es el punto de aplicación del efecto del eje. Por lo tanto $\overrightarrow{O_{m} P_{e j e}}$ es el vector posición del punto de aplicación de las fuerzas del eje sobre la placa desde $\mathrm{O}_{\mathrm{m}}$. Según nuestras consideraciones, suponiendo que el eje se encuentra empotrado en el dicho centro, $\mathrm{O}_{\mathrm{m}}$, este vector de posición será nulo por lo que las fuerzas aplicadas ejercerán un momento de la forma:

$$
\overrightarrow{M_{e j e}}=\overrightarrow{C . G . O_{m}} \wedge\left(\overrightarrow{F_{e j e}}\right)-\overrightarrow{M_{\text {ejeint }}}
$$

\subsubsection{Reacción en los apoyos entre placas}

Siempre que dos placas se encuentren en contacto en una configuración aparecerá una reacción entre ellas. De estas reacciones se conoce que siempre serán verticales y hacia arriba vistas desde la referencia del módulo anterior, tal como indica la figura 9. Pasándola a nuestra base quedará:

$\overrightarrow{F_{\text {reacción }}}=M_{\text {transf base }}\left(00 F_{\text {reacción }}\right)^{T}$

Donde, igual que para el caso de los actuadores, se busca el módulo de la fuerza, $\mathrm{F}_{\text {reacción, }}$ como un valor escalar. En cuanto a $M_{\text {transf base, es la matriz que }}$ lleva $\overrightarrow{F_{\text {reacción }}}$ a nuestra base, solidaria a $\mathrm{O}_{\mathrm{m}}$, igual que en apartado 3.3.2. Dependerá del giro aplicado, dándose los mismos dos casos (5) y (6). Dependiendo 
del caso variarán tanto el valor de reacción como su posición, de manera similar al caso de los actuadores. Las distintas posibilidades serán:

- Casos 1, 2, 4, 8: Movimiento de pivote (figura 9) en el que solo existe una fuerza de reacción en el punto de pivote, situado según la configuración, definido en el vector $\overrightarrow{O_{m} P_{\text {reacclón }}}$ definido en (17).

- Caso 0: Estado de pleno contacto entre placas. Para su estudio suponemos que existen cuatro reacciones iguales distribuidas en cada uno de los puntos de contacto.

- Caso 15: No existe ninguna fuerza de reacción, la configuración se sustenta por los 4 actuadores en conjunto por lo que no hay contacto entre placas y no habrá efecto de reacciones.

Dependiendo del caso se define el vector de aplicación desde $\mathrm{O}_{\mathrm{m}}$ de la siguiente manera:

$\overrightarrow{O_{m} P_{\text {reacclón }}}=\left(\begin{array}{c}0 \\ \pm d_{\text {reacc }} \\ -h_{\text {min }} * 0.5\end{array}\right)$ ó $\left(\begin{array}{c} \pm d_{\text {reacc }} \\ 0 \\ -h_{\text {min }} * 0.50\end{array}\right)$

El vector completo de aplicación será:

$$
\overrightarrow{\text { C.G.P } P_{\text {reacción }}}=\overrightarrow{C . G . O_{m}}+\overrightarrow{O_{m} P_{\text {reacción }}}
$$

Donde $P_{\text {reacción }}$ es el punto donde se ejerce la reacción $\mathrm{y} d_{\text {reacc }}$ la distancia en el eje ' $\mathrm{x}$ ' o ' $\mathrm{y}$ ' del centro $\mathrm{O}_{\mathrm{m}}$ hasta dicho punto. Eligiendo el vector con $d_{\text {reacc }}$ en la componente ' $\mathrm{x}$ ' para las configuraciones 2 y 8 , con su signo correspondiente. Para las configuraciones 1 y 4 se elige el vector con $d_{\text {reacc }}$ en la componente ' $y$ ', también con el signo correspondiente.

El momento respecto a C.G. será:

$$
\overrightarrow{M_{\text {reacción }}}=\overrightarrow{C . G . P_{\text {reacción }}} \wedge \overrightarrow{F_{\text {reacclón }}}
$$

\subsubsection{Efecto del choque entre placas}

Existen ciertos casos, cuando se desactiva un actuador, que su extremo queda en voladizo y avanza hasta chocar con el módulo anterior. Se dará una fuerza de frenado o de choque que aun siendo similar a la de reacción por apoyo, no se define de la misma manera. Será el paso intermedio hacia la fuerza de reacción que habrá en ese punto. Esta fuerza puntual se tratará como la fuerza de reacción explicada en el apartado 3.3.4, de forma que:

$$
\begin{array}{lr}
\overrightarrow{F_{\text {Choque }}}=0 & \text { si } \theta, \beta \text { ó } h \neq \theta_{\text {choque }}, \beta_{\text {choque }} \text { ó } h_{\text {choque }} \\
\overrightarrow{F_{\text {Choque }}}=\overrightarrow{F_{\text {reacción }}} \quad \text { si } \theta, \beta \text { ó } h=\theta_{\text {choque }}, \beta_{\text {choque }} \text { ó } h_{\text {choque }}
\end{array}
$$

\subsubsection{Oposición del medio externo}

Existirán ocasiones en las que el robot se encuentre en un medio que oponga resistencia a sus movimientos, ya sea en un medio fluido como el interior de un cuerpo, o el mismo aire.

En nuestro caso, la superficie de oposición es perpendicular al flujo. Por lo tanto las fuerzas generadas por los esfuerzos cortantes también lo serán y no contribuirán a la resistencia de nuestro elemento. La resistencia será causada solamente por el efecto de la presión, será resistencia de forma en su totalidad. Se expresa esta resistencia según la ley de Stokes, donde podremos ponerla en función de la velocidad del fluido que conocemos:

$$
\mathrm{D}=C_{D}\left(\frac{1}{2} \rho V_{\infty}^{2} A\right)
$$

Ecuación válida para números de Reynolds altos, que será el caso que se considera. También se establece la suposición de flujo incompresible $(\mathrm{M}<0.3)$. Se define cada término de la siguiente manera:

D.- resistencia ejercida en un sólido a la oposición del paso de un fluido.

$\mathrm{C}_{\text {D.- }} \quad$ coeficiente constante que nos da la relación $\frac{\text { Fuerza resistiva }}{\text { Fuerza viscosa }}$.

p.- $\quad$ Densidad del medio.

$\mathrm{V}_{\infty}$.- Velocidad escalar, módulo de la velocidad normal a la superficie de oposición.

A.- $\quad$ Superficie frontal de la última placa. Será la superficie de oposición.

Se calculará la fuerza tan solo si la velocidad es negativa, es decir, si se opone a la superficie y, por lo tanto, es capaz de aplicar la fuerza de oposición. La velocidad $V_{\text {total }}$ será la velocidad utilizada como $\mathrm{V}_{\infty}$. Considerar que al tomar solo la componente en ' $z$ ', se trata de una velocidad escalar, pero dependiente de las variables $\theta, \beta$ y h y sus derivadas. Calculamos a partir de $V_{\text {total }}$ la fuerza media ejercida en la superficie. Por el teorema del valor medio:

$$
\mathrm{D}_{\text {media }}=\frac{C_{D}\left(\frac{1}{2} \rho A\right)}{\operatorname{rmod} \cdot 2 \pi} \int_{0}^{\text {rmod }} \int_{0}^{2 \pi} V_{\text {total }_{k}}{ }^{2} d r_{\text {aux }} d \alpha_{\text {aux }}
$$

Esta será la fuerza de oposición equivalente a la fuerza ejercida por la distribución de velocidades sobre la superficie de oposición.

El punto de aplicación de $\mathrm{D}_{\text {media }}$ se define como: $\overline{o_{\text {final }} P_{\text {opo }}}=\frac{C_{D}\left(\frac{1}{2} \rho A\right)}{\operatorname{rmod} \cdot 2 \pi \cdot D_{\text {media }}} \int_{0}^{r m o d} \int_{0}^{2 \pi} V_{\text {total }}{ }^{2} \cdot \overline{o_{\text {final }} P_{\text {sup }}} d r_{\text {aux }} d \alpha_{\text {aux }}$ 
Añadiendo la distancia del centro de masas de nuestro bloque de estudio al centro de la superficie de oposición, $\overrightarrow{C . G . O_{\text {flnal }}}$, se obtiene:

$$
\overrightarrow{C . G . P_{\text {opo }}}=\overrightarrow{C . G . O_{\text {final }}}+\overrightarrow{O_{\text {final }} P_{\text {opo }}}
$$

Finalmente se obtiene la fuerza y el momento resultantes de la oposicional movimiento. Considerando $\vec{n}$ como el vector unitario de dirección y sentido igual a la velocidad obtenida $\vec{V}_{\text {total }_{k}}$ :

$$
\overrightarrow{F_{\text {opo }}}=\mathrm{D}_{\text {media }} \cdot \vec{n}
$$

Y el momento respecto a C.G. quedará:

$$
\overrightarrow{M o p o}=\overrightarrow{C . G . P_{\text {opo }}} \wedge \overrightarrow{\text { Fopo }}
$$

Para realizar el estudio dinámico, se considera el efecto de todos los esfuerzos explicados en los apartados anteriores en cada placa por separado, aplicando las ecuaciones de momento cinético y la segunda ley de Newton.

Se ha considerado el movimiento del robot módulo a módulo desde una configuración inicial, $\mathrm{M}_{\text {ini }}$, a otra final, $\mathrm{M}_{\text {fin }}$, es decir, no hay dos módulos moviéndose simultáneamente, y consideramos que de la base al extremo.

\section{ANALISIS DE RESULTADOS}

Como material principal en la composición de las placas se ha elegido PVC rígido, con una forma como la de la figura 2, despreciando el espacio que ocupan los actuadores para el cálculo de la masa.

Para configuración del eje se ha tomado la forma tubular con el objetivo de servir de resguardo al cableado además de su función estructural. El material elegido es goma de silicona (SIR).

A continuación se muestran los resultados obtenidos correspondientes en dos casos distintos.

Caso 1: giro

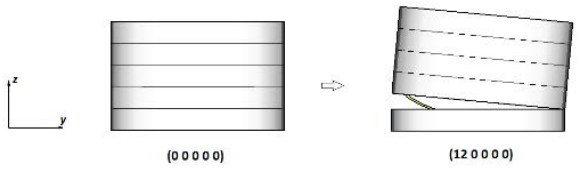

Figura 9: Representación de un giro

Este caso se centrando en el estudio de la fuerza del actuador. Se parte de un robot de 5 módulos, con una configuración inicial $\mathrm{M}_{\mathrm{o}}\left(\begin{array}{lllll}0 & 0 & 0 & 0 & 0\end{array}\right)$ y buscando una configuración final M (12 00000$)$, como se muestra en la figura 9.

A partir de esta configuración se podrá ver la solicitación del único actuador activo. Para ello a esta misma configuración se le aumenta progresivamente el número de módulos superiores, manteniendo el movimiento en el primer módulo.

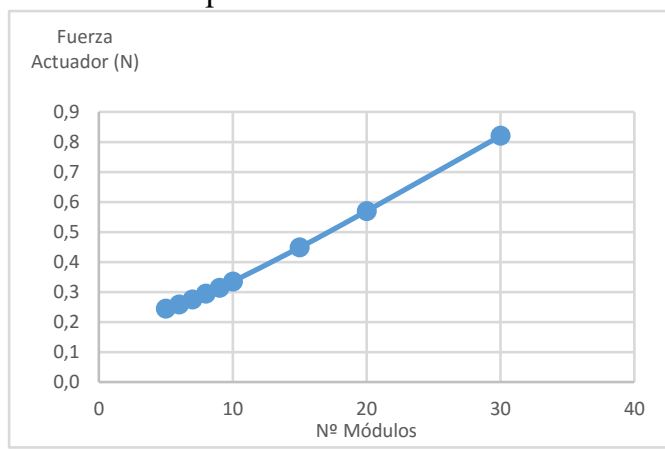

Figura 10: Progresión de las necesidades del actuador en el caso 1 según el número de módulos.

En la figura 10 se puede observar la progresión de la fuerza necesaria en el actuador según la cantidad de módulos que conformen el robot. Pasando de 0,2441 $\mathrm{N}$ con 5 módulos, para un robot significativamente corto, hasta $0,8199 \mathrm{~N}$ para 30 módulos, teniendo en cuenta que el peso estimado por módulo se sitúa sobre los 4,8 gramos. En la tabla 2 se muestran los incrementos en dicha fuerza relacionados con los incrementos en el número de módulos:

Tabla 2: Comparación de resultados en el caso 1.

\begin{tabular}{|c|c|c|c|}
\hline $\begin{array}{c}\text { Casos } \\
\text { comparados }\end{array}$ & $\begin{array}{c}\Delta \mathbf{F}_{\text {actuador }} \\
(\%)\end{array}$ & $\begin{array}{c}\Delta \mathbf{n}^{\mathbf{0}} \mathbf{d e} \\
\mathbf{m o ́ d u l o s} \\
\mathbf{( \% )}\end{array}$ & $\frac{\Delta \mathbf{F}_{\text {actuador }}}{\Delta \mathbf{n}^{\circ} \text { de módulos }}$ \\
\hline $\begin{array}{c}5 \text { mód. } \rightarrow \\
10 \text { mód. }\end{array}$ & 137.16 & 2 & 68.6 \\
\hline $\begin{array}{c}10 \text { mód. } \rightarrow 15 \\
\text { mód. }\end{array}$ & 133.84 & 1.5 & 89.2 \\
\hline $\begin{array}{c}15 \text { mód. } \rightarrow 20 \\
\text { mód. }\end{array}$ & 127.8 & 1.33 & 95.3 \\
\hline $\begin{array}{c}20 \text { mód. } \rightarrow 30 \\
\text { mód. }\end{array}$ & 143.97 & 1.5 & 95.9 \\
\hline
\end{tabular}

El incremento de la fuerza solicitada comparado con el incremento en el número de módulos no se mantiene constante. Para una cantidad pequeña de módulos el efecto del peso pierde relevancia respecto al efecto del eje. Para cantidades mayores el incremento de la fuerza del actuador según el incremento de módulos es mayor y se mantiene constante. Esto refleja una mayor importancia del efecto del peso de los módulos.

\section{Caso 2: traslación}

Buscando estudiar la rigidez del eje cambiamos la orientación de la base del robot, y elegimos un cambio de configuración de $\mathrm{M}_{\mathrm{o}}(015151515)$ a M (15 1515 15 15) según la figura 11.

Los resultados obtenidos se muestran en la figura 12 . A partir de cierto número de módulos los valores de fuerza solicitada a cada actuador son negativos. Físicamente, el actuador debería ser capaz de ejercer una fuerza de atracción entre placas, en vez de repulsión, para resolver el sistema que le hemos 
impuesto, lo que no es posible según la definición de los actuadores IPMC.

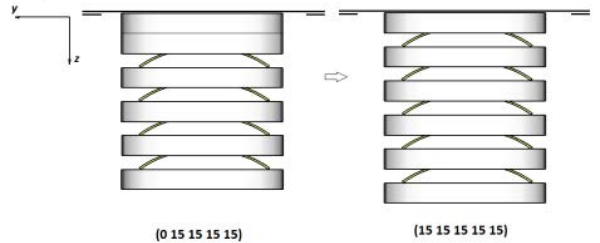

Figura 11: Representación de una translación.

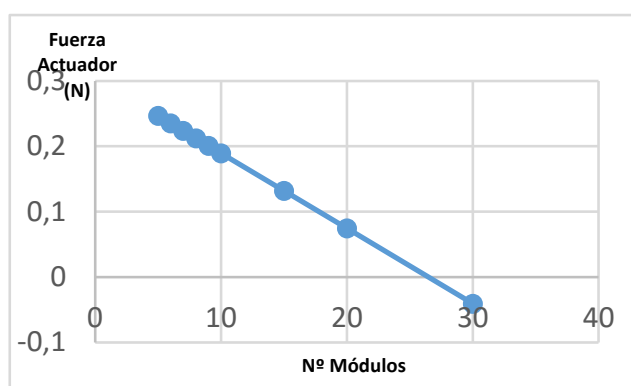

Figura 12: Progresión de las necesidades del actuador.

Esto nos indica que o bien por la morfología o por el material del eje no es capaz de soportar los esfuerzos a los que se le somete. Tendríamos un fallo por la parte estructural el eje.

\section{CONCLUSIONES}

Según las dimensiones del robot ápodo, se elegiría una longitud para el actuador de $10 \mathrm{~mm}$. Comprobando en la gráfica, un IPMC de esa longitud podría llegar a dar 2,5 gf, que son $0,0245 \mathrm{~N}$. Retomando los datos que se han obtenido en el apartado anterior en la configuración más desfavorable se tiene en torno a 0,9 $\mathrm{N}$ de fuerza necesaria en el actuador. Es decir, la fuerza necesaria sería del orden de diez veces la que podría suministrar el IPMC. A primera vista el actuador elegido resultaría insuficiente. Se deberán buscar soluciones.

Una solución podría consistir montar varios IPMC en paralelo. El principal inconveniente de esta solución sería el espacio, dado que se busca minimizar el robot lo máximo posible, tanto en peso como en volumen. Por otro lado, puede buscarse disminuir las solicitudes del actuador mediante la elección de materiales más livianos que consigan mantener rígido el sistema, y la elección de un eje más flexible. La tercera solución y quizá la más factible sería elegir otro tipo de actuador. Un posible actuador alternativo sería el actuador de polímeros conductivos [7], cuyo funcionamiento se basa en reacciones redox y que posee una mayor potencia específica que los IPMC. Este incremento de potencia, en torno a una fuerza diez veces mayor a la aportada por los IPMCs, junto con una reducción del peso podría asegurar la funcionalidad del sistema.

\section{Agradecimientos}

Este trabajo ha sido financiado por el Ministerio de Economía y Competitividad, dentro del proyecto coordinado MARCUS Micro Abdominal Robot Cooperative United System. DPI2013-47196-C3-1-R.

\section{Referencias}

[1] Carreira Pinheiro E. Análisis cinemático y planificación de movimientos de un robot endoscópico accionado electromagnéticamente. Instituto Politecnico de Bragança, 2011.

[2] Cosentino F., Tumino E., Rubis Passoni G., Rigante A., Barbera R., Tauro A., Cosentino P. E. Robotic Colonoscopy. Colonoscopy, Ed. InTech, 2011, 291-308.

[3] Garg A., Vikram C. S., Gupta S., Sutar M. K., Pathak P. M., Mehta N. K., Gupta V. K. Design and development of In Vivo Robot for biopsy. Mechanics Based Design of Structures and Machines 2014, 42: 278-295.

[4] Garzón Oviedo M. A. Estrategias Bio-Inspiradas para Locomoción de Robots Ápodos. Trabajo Fin de Máster de la Escuela Técnica Superior de Ingenieros Industriales, Universidad Politécnica de Madrid, 2011.

[5] Kwok K.-W., Tsoi K. H., Vitiello V., Clark J., Chow G. C. T., Luk W., Yang G.-Z. Dimensionality Reduction in Controlling Articulated Snake Robot for Endoscopy Under Dynamic Active Constraints. Transactions of Robotics. IEEE 2012, 1-17.

[6] Liu X., Tavanapong W., Wong J., Oh J., de Groen P. C. Automated measurement of quality of mucosa inspection for colonoscopy. Proceding Computer Science 2012, 1:951-960.

[7] Madruga S. Análisis de tecnologías y materiales para el diseño de un robot ápodo, modular e híper-redundante, Universidad de Valladolid, 2015.

[8] Muñoz S. Análisis de la cinemática inversa y minimización de errores mediante algoritmos de optimización para un robot hiper-redundante. Universidad de Valladolid, 2013.

[9] Osama A., Solieman H.H., Zaytoun H.A. Role of CT virtual colonoscopy versus conventional colonoscopy in the evaluation of colonic polyps. The Egyptian Journal of Radiology and Nuclear Medicine 2013, 44:425-432.

[10] Palmer D., Cobos-Guzmán S., Axinte D. Realtime method for tip following navigation of continuum snake arm robots. Robotics and Autonomous Systems 2014, 62: 1478-1485.

[11] Pedrejón, E. Estudio dinámico del comportamiento de un robot ápodo, modular e híper-redundante, Universidad de Valladolid, 2016. 